treatment and at least once again after the treatment. Anakinra $100 \mathrm{mg}$ daily was administered for 1 to 3 consecutive days. Other baseline demographic data and long-term follow up data were collected from the patients' charts and after phone call to the patients

Results: 13 patients fulfilled the inclusion criteria. 12 had a flare at the shoulder, 1 at the first MCP of the hand. The acute inclusion flare was the first episode in 9/13 subjects. At baseline, mean pain VAS:8.8/10 Active and passive motion was severely restricted in all patients. Local inflammation was sufficiently severe to induce an elevation of the CRP and ESR in 10/12 patients. By ultrasound, the mean size of calcifications was $17 \mathrm{~mm}$. Only one had an arc-shaped aspect with shadowing, the others were fragmented. Short term follow-up: Pain diminished drastically and rapidly, typically within hours, in most patients after the first injection and was almost absent at day three (mean VAS 1.7/10), stable at 3 weeks (mean VAS: 2.1). At follow-up ultrasound, the calcification disappeared in none of the patients but, in most cases, a reduction (50\% mean size reduction) and a fragmentation was observed.Long term follow-up: The median duration was 24 months, (range 4 to 74 months). Only $2 / 12$ patients experienced an acute relapse. These two patients already suffered from chronic shoulder pain and had prior flares before the treatment with anakinra. Mild chronic pain, essentially related to movement (mean VAS: 2.7), was still present in 5 patients. Two patients regularly took NSAIDs; the others did not use any painkiller

Conclusions: This study suggests that IL-1 $\beta$ inhibition may be an interesting therapeutic approach in acute flares in relation with hydroxyapatite calcification. Moreover this treatment does not seem to be associated with rebound or recurrence of flares in the long term.

References:

[1] Zufferey P, So AK.A pilot study of IL-1 inhibition in acute calcific periarthritis of the shoulder Ann Rheum Dis. 2013 Mar;72(3):465-7.

Disclosure of Interest: None declared

DOI: 10.1136/annrheumdis-2017-eular.2730

\section{THU0453 HEART AND CAROTID CHANGES IN FIFTY-THREE GOUT PATIENTS TREATED WITH XANTHINE OXIDASE INHIBITORS: A FOLLOW-UP STUDY}

R.N. Gancheva ${ }^{1,2}$, A. Kundurdjiev ${ }^{1,2}$, M. Ivanova ${ }^{1,2}$, T. Kundurzhiev ${ }^{2,3}$, Z. Kolarov ${ }^{1,2} .{ }^{1}$ Medical Faculty; ${ }^{2}$ Medical University; ${ }^{3}$ Faculty of Public Health, Sofia, Bulgaria

Background: Gout connects to cardiovascular (CV) morbidity and higher risk of death due to CV events. A few ultrasound studies assess the way in which heart and vessels change over time in gout patients (pts). It is still unclear whether treatment with allopurinol or febuxostat reduces to some extent target organ damage.

Objectives: We aimed to establish heart and vessels alterations developing over time in gout pts and to find out whether treatment with allopurinol or febuxostat is associated with a change in these structures.

Methods: A total of 53 gout pts were examined and divided into two groups: 31 gouty arthritis without tophi, 24 males and 7 females aged $55.8 \pm 12.3$ years and 22 gouty tophi, 20 males and 2 females aged $59 \pm 9$ years. Pts underwent multimodal ultrasound examination at study entry and one year and six months thereafter. Aortic root diameter (Ao), left atrium (LA) size, thickness of the interventricular septum (IVS) and of posterior wall (PW) of the left ventricle, end-diastolic volume index (EDVi), end-systolic volume index (ESVi), stroke volume index (SVi), fractional shortening (FS), and ejection fraction (EF) were recorded with 2.5 $\mathrm{MHz}$ transducer. Intima-media thickness (IMT) and rigidity of the common carotid arteries, judged by the values of the common carotid artery resistive index (CCARI), were examined with $10 \mathrm{MHz}$ transducer. Statistical analyzes were done by chi-square or Fisher's exact test, One-Sample Kolmogorov-Smirnov, ShapiroWilk and Paired-Samples T-test. Within-subjects effects and between-subjects effects were assessed by the Repeated Measures ANOVA.

Results: At the two time points of pts' examination there was no deviation in $\mathrm{Hb}$ $(p=0.412)$, Ht $(p=0.866)$, serum uric acid $(p=0.877)$, serum albumin $(p=0.515)$, eGFR ( $p=0.793)$, EDVi $(p=0.248)$, ESVi $(p=0.345)$, SVi $(p=0.357)$, FS $(p=0.516)$ and CCARI $(p=0.244)$, but PW tended to be thicker $(p=0.067)$. Ao $(p=0.008), L A$ $(p=0.001)$, IVS $(p=0.007)$ increased, and EF $(p=0.026)$ decreased between the two examinations. In gouty arthritis without tophi and in gouty tophi Ao (mean $\pm S D$; $39.42 \pm 5.00$ vs $39.14 \pm 3.77, p=0.471$ ), LA (mean $\pm S D ; 39.10 \pm 5.11$ vs $43.86 \pm 5.95$, $\mathrm{p}=0.088$ ), IVS (mean $\pm S D ; 12.77 \pm 1.58$ vs $13.47 \pm 1.54, \mathrm{p}=0.910$ ) and IMT (mean $\pm S D ; 0.93 \pm 0.17$ vs $1.05 \pm 0.17, p=0.237$ ) changed similarly over time, but the reduction in EF was more pronounced in gouty tophi $(p=0.031)$. In pts not taking allopurinol an increase in LA $(p=0.012)$, IVS $(p=0.006)$ and IMT $(p<0.001)$ was registered. In those treated with allopurinol Ao $(p=0.011)$, LA $(p=0.022)$ increased, EF $(p=0.016)$ decreased and no change in IVS $(p=0.523)$ and IMT $(p=0.165)$ was found. Pts who had not received febuxostat tended to have greater $A o(p=0.063)$, larger LA $(p=0.003)$, thicker IVS $(p=0.046)$, thicker IMT $(p<0.001)$ and lower EF $(p=0.034)$. In contrast, no change was registered in Ao $(p=0.559), L A(p=0.332)$, IVS $(p=0.125)$, EF $(p=0.689)$ and IMT $(p=0.163)$ in treated with febuxostat.

Conclusions: Over time in gouty arthritis without tophi and gouty tophi similar changes in the heart and carotids develop, but heart pumping function is more affected in the later stage of the disease. Heart and carotids morphology and function are preserved in pts treated with febuxostat. In the allopurinol group cardiac morphological and functional alterations occur with no change in IMT.
Disclosure of Interest: None declared

DOI: 10.1136/annrheumdis-2017-eular.2516

\section{THU0454 ROLE OF PHYSICAL EXERCISE IN PATIENTS WITH HIP ARTHROPLASTY FOR ASEPTIC NECROSIS OF THE FEMORAL HEAD}

R.I. Marcu, S. Patru, A.C. Bighea, D. Matei. Medical Rehabilitation, University of Medicine and Pharmacy, Craiova, Romania

Background: Necrosis of the femoral head has a slow but progressive evolution, increasing degradation of the joint, worsening instability, with permanent pain and reduced walk perimeter. In the advanced stages of the disease, the only effective treatment is replacement of the joint (hip arthroplasty).

Objectives: The objective of our study was to examine the effects of a two weeks kinetic program on pain and functional status in patients with hip arthroplasty for aseptic necrosis of the femoral head.

Methods: We conducted an observational, prospective, randomized study on a sample of 47 patients with hip arthroplasty for aseptic necrosis of the femoral head. Patients were randomly assigned to a control group (23 patients) who received only analgesics and NSAID's when needed (group 1) and a study group (24 patients) whose therapeutic program included daily physical exercise (group 2). Patients were initially evaluated and after two weeks of rehabilitation treatment. The clinical and functional parameters assessed were: pain on a visual analogue scale (100 mmVAS), physical impairments (muscular strength and mobility of hip joint) and disabilities (Tinetti Gait Scale, D'Aubigné Scale and movement capacity).

Results: The scores for functional parameters improved: pain- 42.6\% (group 2) and $33.4 \%$ (group 1) ( $\mathrm{p}=0.000054)$; physical impairments: muscular strength$8.3 \%$ (group 2), without improving by group 1, mobility: $41.8 \%$ (group2) and $21.4 \%$ (group 1); disabilities: Tinetti Gait Scale- 33.6\% (group 2) and $22.1 \%$ (group 1), D'Aubigné Scale- $35.8 \%$ (group 2) and $22.4 \%$ (group 1), movement capacity$55.8 \%$ (group2) and $31.9 \%$ (group 1). The results were statistic significant $(\mathrm{p}<0.05)$.

Conclusions: Improvement of pain, physical impairments and disabilities for the study group certifies the efficacy of the rehabilitation program including physical exercise in patients with hip arthroplasty for aseptic necrosis of the femoral head and motivates the continuation of the study on a longer period of time and on a larger number of patients. Rehabilitation program must begin right after patients underwent surgery for hip replacement.

Disclosure of Interest: None declared

DOI: 10.1136/annrheumdis-2017-eular.5130

\section{THU0455 RENAL SAFETY OF LESINURAD: A POOLED ANALYSIS OF PHASE III AND EXTENSION STUDIES}

R. Terkeltaub ${ }^{1}$, R. Malamet ${ }^{2}$, K. Bos ${ }^{2}$, J. Li ${ }^{2}$, D.S. Goldfarb ${ }^{3}$, M. Pillinger ${ }^{4}$, D. Jalal ${ }^{5}$, J. Hu ${ }^{6}$, K. Saag ${ }^{7} .{ }^{1}$ University of California, San Diego; ${ }^{2}$ AstraZeneca Pharmaceuticals, Wilmington; ${ }^{3}$ New York University School of Medicine; ${ }^{4}$ New York University Langone Medical Center, New York; ${ }^{5}$ University of Colorado Anschutz Medical Center, Aurora; ${ }^{6}$ Formerly Ardea Biosciences, Inc, San Diego; ${ }^{7}$ University of Alabama at Birmingham, Birmingham, United States

Background: Lesinurad (LESU) is a selective uric acid reabsorption inhibitor approved in the United States and European Union at a $200 \mathrm{mg}$ daily dose in combination with a xanthine oxidase inhibitor (XOI) for treatment of hyperuricemia associated with gout in patients unable to achieve target serum uric acid on an XOI (allopurinol or febuxostat) alone.

Objectives: To investigate the renal safety of LESU, data were pooled from 3 pivotal, placebo-controlled, 12-month phase III (core) studies evaluating lesinurad $200 \mathrm{mg}$ (LESU200) and $400 \mathrm{mg}$ (LESU400) in combination with an XOI and from 2 extension studies, where patients continued LESU+XOI at the same dose or were randomized from placebo to LESU200 or LESU400 plus an XOI.

Methods: Renal-related and kidney stone safety data were pooled from core studies to compare LESU200+XOI and LESU400+XOI with an XOI alone and from core studies + extension studies to evaluate the impact on renal safety of extended LESU+XOI treatment. Renal-related treatment-emergent adverse events (TEAEs) were a customized list of 36 preferred terms selected from the Medical Dictionary for Regulatory Activities (MedDRA) Renal and Urinary Disorders System Organ Class (SOC), the Investigations SOC, and the Acute Renal Failure MedDRA Standardized MedDRA Query (SMQ). Descriptive statistics are provided for patients receiving $\geq 1$ dose of study medication. To adjust for varying treatment duration, TEAEs are expressed as exposure-adjusted incidence rates (EAIRs; number of subjects with events per 100 person-years).

Results: In the core studies, EAIRs for any renal-related TEAE, serious renalrelated TEAEs, and renal-related TEAEs leading to discontinuation were similar with an XOI alone and LESU200+XOI and lower than with LESU400+XOI (Table 1). Similar results were found for kidney stone and serious kidney stone TEAEs. The most common renal-related TEAE was increased serum creatinine $(\mathrm{sCr})$. EAIRs for $\mathrm{sCr}$ elevations $\geq 1.5 \mathrm{x}$ baseline were higher with LESU+XOI than XOI alone (Table 1). Overall, $75 \%$ and $84 \%$ of $\mathrm{sCr}$ elevations in the $\mathrm{XOI}$ alone and LESU+XOI groups, respectively, were resolved at last study assessment; $75 \%$ and $66 \%$ resolved without interruption of medication. Exposure to extended 